\title{
Methodologic Approaches to Histologically Distinguishing Vaccine versus Nonvaccine-Associated Sarcomas Using Validated Time and Location Vaccination Histories in Cats
}

\author{
Anup Srivastav, ${ }^{1}$ Philip H. Kass, ${ }^{1}$ Lawrence D. McGill, ${ }^{2}$ \\ Michael S. Kent, ${ }^{3}$ and Thomas B. Farver ${ }^{1}$ \\ ${ }^{1}$ Department of Population Health and Reproduction, School of Veterinary Medicine, University of California, Davis, CA 95616, USA \\ ${ }^{2}$ Animal Reference Pathology Division, ARUP Laboratories, Salt Lake City, UT 84108, USA \\ ${ }^{3}$ Department of Surgical and Radiological Sciences, School of Veterinary Medicine, University of California, Davis, CA 95616, USA
}

Correspondence should be addressed to Philip H. Kass, phkass@ucdavis.edu

Received 24 May 2011; Accepted 11 July 2011

Academic Editors: B. Azadeh and A. Stringer

Copyright (c) 2011 Anup Srivastav et al. This is an open access article distributed under the Creative Commons Attribution License, which permits unrestricted use, distribution, and reproduction in any medium, provided the original work is properly cited.

\begin{abstract}
Epidemiologic studies of feline vaccine-associated sarcoma (FVAS) require accurate sarcoma case definitions free from unverified assumptions for valid causal inference. This study developed methodologic approaches to improve reliable differentiation of vaccine versus nonvaccine-associated sarcomas on histologic characteristics using time windows of vaccine histories and body geographic information. Four case and five control definitions were used to classify 124 sarcomas. Results showed presence in the subcutis versus dermis, increased nuclear pleomorphism, more granulation tissue, and higher presence of inflammation in FVAS than presumptive non-FVAS. Correctly classifying sarcomas using stringent classification criteria as employed in this study will help reduce misclassification of FVAS in future epidemiologic studies of comparative risk.
\end{abstract}

\section{Introduction}

Although twenty years have elapsed since the initial recognition of feline vaccine-associated sarcoma (FVAS), this iatrogenic malignancy remains a pervasive problem to cat owners and veterinarians [1]. Although considerable research has been conducted to better define the determinants of these tumors either in individual cats [2,3] or populations [4-7], and despite changes in vaccine formulation and administration strategies over the last 15 years, cases of FVAS continue to be reported. Although this iatrogenic condition has not been reproduced in experimental studies due to its rarity [8], epidemiologic research has convincingly demonstrated a causal relationship, particularly with respect to rabies and feline leukemia virus vaccines, and the veterinary medical and vaccine manufacturing communities have taken steps to try to ameliorate incidence. Earlier work using statistical models to compare histologic characteristics of presumptive feline vaccine-associated sarcomas and presumptive nonFVAS implicitly assumed that a sarcoma was vaccine induced if the sarcoma arose at a site conventionally used for vaccination in the cat $[9,10]$. However, no previously published studies had documented verification of the patients' actual vaccine histories, that is, precisely when and where the vaccine was locally administered (if at all) relative to onset of tumor development and tumor location, respectively. It is unjustified to assume that a sarcoma is vaccine associated because it occurred at a commonly used vaccination site. Stringent classification criteria should advisedly include documentation of a reasonably accurate vaccination location, date of vaccination, and precise body location where the sarcoma developed. A sarcoma can only be defined as vaccine associated if it develops at or near a vaccination site following a biologically plausible induction interval. This underscores both the importance and subtleties of unambiguously specifying and defining an FVAS. For example, how close should a tumor be to a vaccination site to be associated? What is a biologically plausible induction interval following vaccination? Such nonidentifiability makes the extent of misclassification in earlier publications impossible to know, 
but careful consideration of these issues can potentially minimize misclassification of FVAS diagnoses and vaccine exposures.

Earlier studies comparing histological differences between presumptive FVAS and non-FVAS suggested that the former are distinguished by one or more of the following: increased nuclear, cellular pleomorphism, mitotic activity, multinucleated giant cells, inflammatory peritumoral infiltration consisting of lymphocytes and/or macrophages and/or neutrophils, myofibroblastic differentiation, substantial necrosis, subcutaneous locations, and variability in density of extracellular matrix [9-11].

In the absence of such stringent criteria, it remains tenuous for pathologists to convincingly and reliably distinguish an FVAS from a non-FVAS. However, it is possible using such criteria to create mathematical models to reinforce pathologists' visual and microscopic assessment and classification of these sarcomas as vaccine associated (or not). Besides helping to correctly characterize the sarcomas, such models could contribute to future epidemiologic studies of risk factors and incidence by diminishing their misclassification.

The goal of this study was to better understand and characterize histologic differences between a vaccine-associated and nonvaccine-associated sarcoma using a stringent set of classification criteria, time windows of vaccine histories, and body geographic information, and to develop study design and statistical methods for distinguishing these two etiologically different sarcoma types.

\section{Methods}

2.1. Case and Control Selection and Definition. A computer search of feline necropsy and biopsy case records from January 1, 2005 through December 31, 2006 was undertaken at Animal Reference Pathology Inc. (ARUP), Salt Lake City, Utah. Cases of the following types of soft tissue (i.e., nonbone) located sarcoma were defined as eligible for inclusion: fibrosarcoma $(n=102)$, malignant fibrous histiocytoma, myxofibrosarcoma $(n=7)$, poorly differentiated sarcoma $(n=1)$, spindle cell sarcoma $(n=0)$, round cell sarcoma $(n=0)$, myofibroblastic sarcoma $(n=0)$, rhabdomyosarcoma $(n=0)$, leiomyosarcoma $(n=0)$, and undifferentiated sarcoma $(n=4)$. Other tumors that have been associated with this phenomenon were also included such as osteosarcoma $(n=6)$, chondrosarcoma $(n=1)$, hemangiosarcoma $(n=1)$, liposarcoma $(n=1)$, and lymphoma $(n=1)$. Information accompanying the biopsy samples from each accession was retrieved that included age, breed, sex, and referring veterinarian contact information. A total of 124 sarcomas were identified for this study.

Materials pertinent to this research were then submitted to the referring veterinarian within three months of tumor diagnosis. These included a survey with detailed vaccination and tumor history questions, a topographical coordinate map with grids of ventral and dorsal views of a cat (Figure 1), and a list of commercially available vaccines. The map was included as guide to allow veterinarians to mark with precision and accuracy the sites of vaccination and sarcoma development.

In addition to characterizing sites by coordinates, conventionally used vaccination sites were defined as interscapular/scapular, flank/paralumbar, dorsolateral thorax, dorsal back, neck, femoral regions, distal limbs, and tail [12] to compare this study's findings to those of earlier publications. Nonvaccination sites were defined as any other location not conventionally used for vaccination.

Four case definitions were used for this study. Case Group 1 was defined as cats with a documented history of vaccination time no less than 30 days to no greater than one year of tumor histopathology injected within one grid on any side of the tumor site $(n=15)$. Similarly, Case Group 2 had a history of vaccination no less than 30 days to no greater than two years $(n=27)$; case Group 3 had a history of vaccination no less than 30 days to no greater than three years $(n=34)$; case Group 4 had a documented history of vaccination at sites commonly used for vaccination regardless of vaccine history at tumor site $(n=87)$. Cases can belong to more than one case definition group.

Five control definitions were used for this study. Control Group A was defined as cats with a documented history of vaccination no less than 365 days injected within one grid on any side of the tumor site $(n=18)$. Similarly, control Group B had a documented history of vaccination no less than 730 days $(n=8)$; control Group $C$ as cats whose tumors arose at sites typically used for vaccination and with no history of vaccination at tumor site within the previous three years provided by veterinarians $(n=15)$; control Group D as cats whose tumors arose at sites not typically used for vaccination and with verification that they were not previous vaccination sites $(n=35)$; control Group E as cats whose tumors developed at sites not typically used for vaccination even if no vaccine history was available $(n=37)$. Controls can belong to more than one control definition group.

2.2. Histopathological Examination. Tissue sections of sarcomas previously evaluated by an ACVP board-certified pathologist at ARUP were reexamined by a one of four pathologists blinded to tumor location and vaccination history. Histological features examined are shown in Table 1.

2.3. Statistical Analysis. Case and control cats' histological tumor characteristics were compared using exact logistic regression (LogXact-8, Cytel Software Corp., Cambridge, Mass). Continuous variables were verified to have log-linear relationships with the outcomes. Characteristics associated with vaccine-site sarcomas in univariate analyses $(P \leq 0.20)$ and patient age (as a potential confounder) were included in multivariate models and retained if their $P$ values $\leq 0.20$. Results are presented as odds ratios (OR), 95\% confidence intervals $(95 \% \mathrm{CI})$, and $P$ values. $P$ values $\leq 0.05$ were considered statistically significant.

\section{Results}

The response proportion for the surveys sent to veterinarians was $32.5 \%$ (343 out of 1054). 


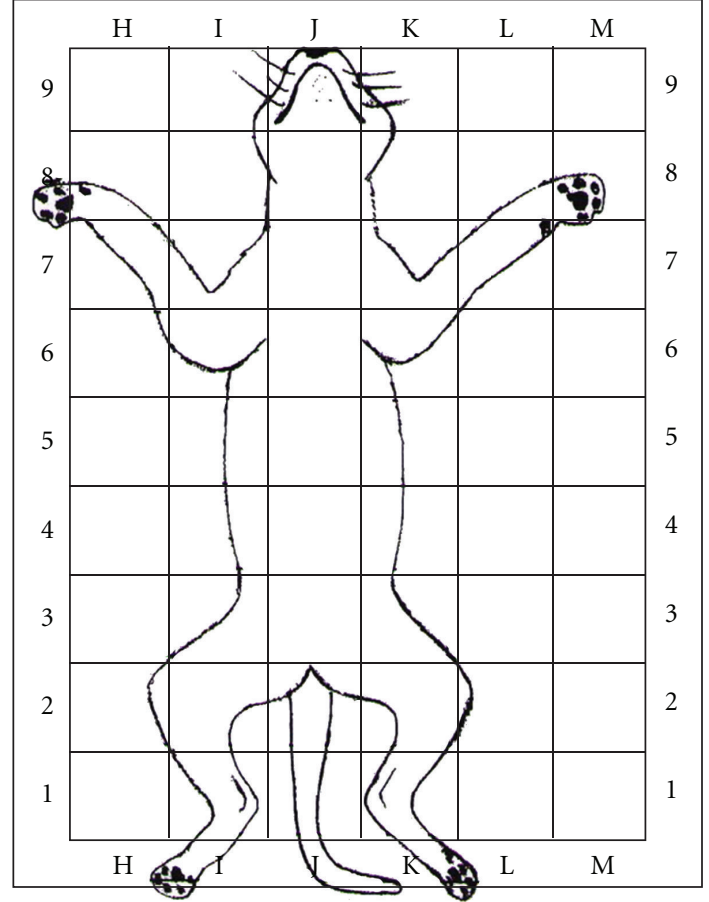

(a)

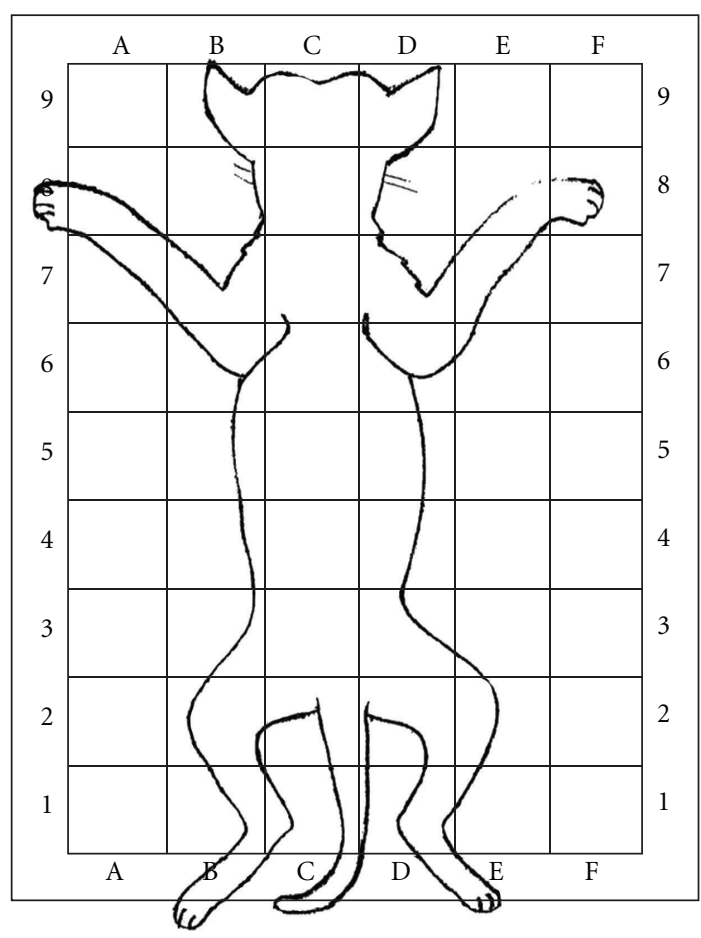

(b)

FIGURE 1: Topographical coordinate grid map illustrating dorsal and ventral views of a cat.

3.1. Sarcomas Arising within One Year of Vaccination. Ageadjusted multivariate logistic regression (Table 2) revealed case Group 1 tumors tended to have lower mitotic indices $(P=0.053)$, less demarcated borders $(P=0.18)$, and less differentiation $(P=0.15)$ than control Group A tumors; and lower mitotic indices $(P=0.090)$ and less nuclear pleomorphism $(P=0.15)$ than control Group B tumors. Case Group 1 tumors were less likely to be located in the dermis $(P<0.0001)$, tended to have increased inflammation $(P=0.081$ and 0.056 , resp. $)$, and had higher numbers of multinucleated giant cells $(P=0.11$ and 0.094 , resp. $)$ than control Group D and E tumors.

3.2. Sarcomas Arising within Two Years of Vaccination. The results of age-adjusted multivariate logistic regression model (Table 2) indicated that case Group 2 sarcomas had significantly lower mitotic indices $(P=0.026)$ and had a tendency to have less nuclear pleomorphism $(P=0.089)$ and decreased neutrophils $(P=0.069)$ compared to control Group B sarcomas. Case Group 2 sarcomas were significantly less likely to be present in the dermis $(P<0.001)$ and had increased myxomatous stromal components $(P=0.002)$ compared to control Group D sarcomas. Compared to control Group E sarcomas, case Group 2 sarcomas were less likely to be present in the dermis $(P<0.001)$, had increased myxomatous stromal components $(P=0.041)$, and had a tendency to have increased inflammation $(P=0.19)$.

3.3. Sarcomas Arising within Three Years of Vaccination. Case Group 3 tumors had a tendency to have higher nuclear pleomorphism $(P=0.17)$ compared to control Group $C$ tumors (Table 2). Compared to control Group D tumors, case Group 3 tumors were less likely to be located in dermis $(P<0.0001)$, had a higher presence of myxomatous stromal components $(P=0.047)$, and had a tendency to have increased inflammation $(P=0.088)$. Case Group 3 sarcomas compared to control Group E sarcomas were less likely to be located in the dermis $(P<0.001)$, had a tendency to have increased inflammation $(P=0.052)$, and had a higher presence of myxomatous stromal components $(P=0.051)$.

3.4. Sarcomas Arising after Vaccination at Traditional Vaccination Sites. Comparing case Group 4 sarcomas to control Group D sarcomas indicated that the former had increased inflammation $(P=0.013)$, was less likely to be dermal $(P<0.0001)$, had increased presence of myxomatous stromal components $(P=0.010)$, and tended to have increased multinucleated giant cells $(P=0.17)$ and diminished neutrophils $(P=0.066)$ (Table 2$)$. Case Group 4 tumors, compared to control Group E tumors, had increased inflammation ( $P=0.023)$, were less likely to be dermal $(P<0.0001)$, had an increased presence of myxomatous stromal components $(P=0.009)$, and tended to have an increased multinucleated giant cells $(P=0.19)$.

\section{Discussion}

This study was conducted to develop and demonstrate methods for designing future studies to distinguish these two etiologically distinct sarcomas. It is the first study to 
TABle 1: Comparison of present study with previously published studies $[9,10]$ on histological characteristics common to all studies distinguishing presumptive vaccine and nonvaccine-associated sarcomas. The number of cases varies by control group category. Statistical differences were denoted by $-(P>0.20),+(P<0.20)$, $++(P<0.05)$, and $+++(P<0.01)$. For previously published studies, $(\mathrm{V})$ indicates characteristic was more common in vaccine-associated sarcomas; (NV) indicates characteristic was more common in nonvaccine-associated sarcomas; (U) indicates could not be determined from publication.

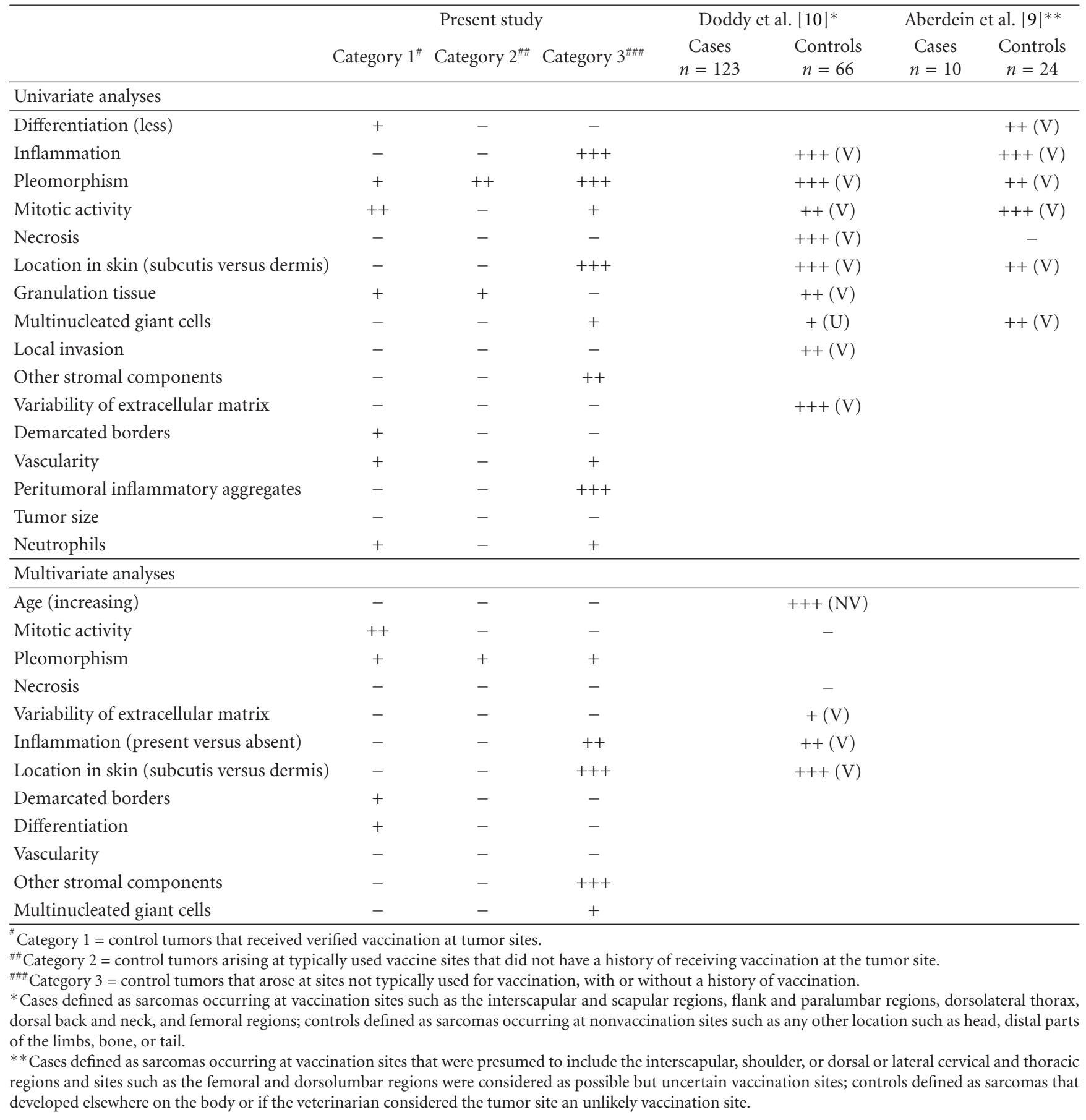

utilize both multivariate statistical methods and detailed histories of vaccination and injection site/tumor location. We approached this problem in a novel way by examining time windows of vaccination history and using a map for diligence in applying stricter exposure definitions. The five control groups can be divided into three tumor categories
(Table 1): (1) received vaccination at the tumor sites; (2) typically used vaccine sites without a history of vaccination there; (3) sites not typically used for vaccination, with or without vaccine history. Distinguishing results were obtained from comparison of cases with the first category of controls: cases showed less neutrophils, mitotic indices, demarcated 


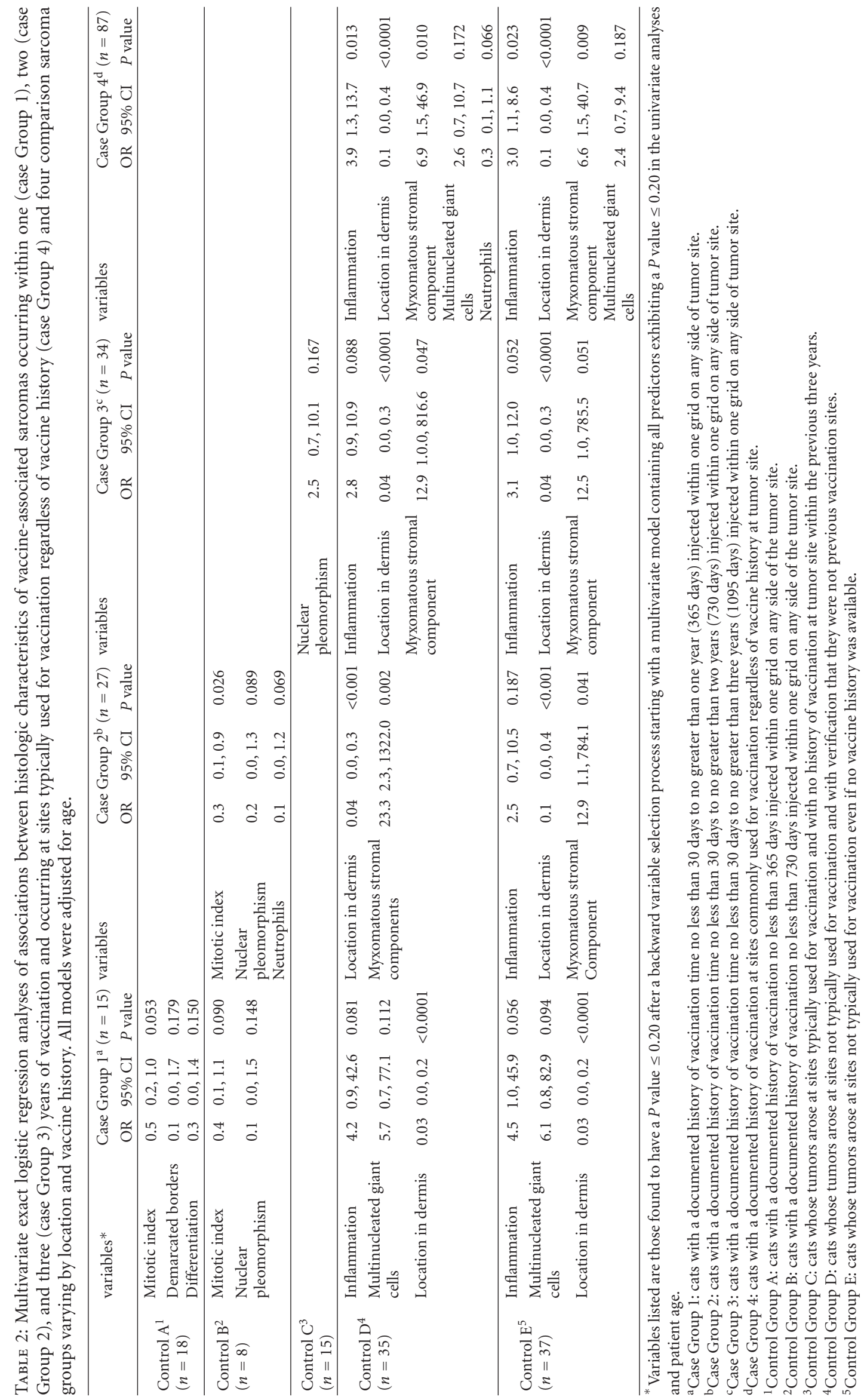


borders, nuclear pleomorphism, and differentiation. These findings are in contrast with earlier statistical studies [9, 10] where presumptive FVAS had higher mitotic indices, increased nuclear pleomorphism, more granulation tissue, and higher presence of inflammation than presumptive nonFVAS. These differences are unsurprising insofar as this category of controls was used to distinguish FVAS that differed only with respect to the timing of their known exposure(s) to vaccines at the tumor sites, suggesting that sarcomas arising sooner following vaccination appear more inflammatory and polymorphic, but not uniformly more anaplastic. Compared to category 2 controls, some cases' (Groups 2 and 3) tumors were characterized by higher nuclear pleomorphism. These findings were consistent with those reported in earlier statistical studies $[9,10]$, although, unlike them, significant differences in mitotic index and inflammation were unobserved. Contrasting the case tumors to category three controls, some results were consistent with earlier findings $[9,10]$. Analyses showed case tumors exhibited increased inflammation, enhanced presence of multinucleated giant cells and myxomatous matrix, diminished presence of neutrophils, and were less likely to be dermal.

Comparing the cases with different time windows of vaccine histories to these controls allowed consistent patterns of histologic case-control differences to emerge. Although the sample sizes for some of the group comparisons were inadequate (and hence some analyses were underpowered) to make confident definitive (versus tentative) conclusions about all histologic feature/sarcoma associations presented, 95\% confidence intervals were provided to demonstrate such imprecision and complement the odds ratios presented.

\section{Conclusions}

Limitations of this study included potentially incorrect recall of vaccine type and administration site. We attempted to mitigate this by enrolling cats prospectively, decreasing the time between tumor diagnosis and data acquisition. Although veterinarians may have been unable to recall the exact location of a vaccine injection if unrecorded in the medical record, we utilized a topographic grid to obtain more accurate information than would have otherwise been possible. Although owners could elect to obtain different vaccines from different veterinarians, we asked veterinarians about vaccines not only administered by them, but by other veterinarians as well. Nevertheless, such information would have to be provided by owners, and due to confidentiality, we were unable to contact them for verification. Despite these limitations, the study's focus on the methodology surrounding the definitions of cases and controls should enfranchise researchers studying the spectrum of issues of FVAS, so that future epidemiologic studies examining additional risk factors or incidence with a diminished probability of classification error may be more confidently accomplished.

\section{Conflict of Interests}

The authors declare that there is no conflict of interests.

\section{Acknowledgments}

The authors gratefully acknowledge the financial support of the George and Phyllis Miller Feline Health Fund, Center for Companion Animal Health, School of Veterinary Medicine, University of California, Davis for this research. The authors also gratefully acknowledge Dr. Glen Esplin, Dr. Gordon Hollinger, and Dr. Sharon Wilson for their help and invaluable assistance in interpreting the pathology.

\section{References}

[1] M. J. Hendrick and M. H. Goldschmidt, "Do injection site reactions induce fibrosarcomas in cats?" Journal of the American Veterinary Medical Association, vol. 199, no. 8, p. 968, 1991.

[2] M. M. G. De Man and R. V. Ducatelle, "Bilateral subcutaneous fibrosarcomas in a cat following feline parvo-, herpes- and calicivirus vaccination," Journal of Feline Medicine and Surgery, vol. 9, no. 5, pp. 432-434, 2007.

[3] R. R. Dubielzig, K. L. Hawkins, and P. E. Miller, "Myofibroblastic sarcoma originating at the site of rabies vaccination in a cat," Journal of Veterinary Diagnostic Investigation, vol. 5, no. 4, pp. 637-638, 1993.

[4] D. G. Esplin, L. D. McGill, A. C. Meininger, and S. R. Wilson, "Postvaccination sarcomas in cats," Journal of the American Veterinary Medical Association, vol. 202, no. 8, pp. 1245-1247, 1993.

[5] M. J. Hendrick, M. H. Goldschmidt, F. S. Shofer, Y. Y. Wang, and A. P. Somlyo, "Postvaccinal sarcomas in the cat: epidemiology and electron probe microanalytical identification of aluminum," Cancer Research, vol. 52, no. 19, pp. 5391-5394, 1992.

[6] P. H. Kass, W. G. BArnes, W. L. Spangler, B. B. Chomel, and M. R. Culbertson, "Epidemiologic evidence for a causal relation between vaccination and fibrosarcoma tumorigenesis in cats," Journal of the American Veterinary Medical Association, vol. 203, no. 3, pp. 396-405, 1993.

[7] P. H. Kass, W. L. Spangler, M. J. Hendrick et al., "Multicenter case-control study of risk factors associated with development of vaccine-associated sarcomas in cats," Journal of the American Veterinary Medical Association, vol. 223, no. 9, pp. 12831292, 2003.

[8] G. M. Gobar and P. H. Kass, "World Wide Web-based survey of vaccination practices, postvaccinal reactions, and vaccine siteassociated sarcomas in cats," Journal of the American Veterinary Medical Association, vol. 220, no. 10, pp. 1477-1482, 2002.

[9] D. Aberdein, J. S. Munday, C. B. Dyer, C. G. Knight, A. F. French, and I. R. Gibson, "Comparison of the histology and immunohistochemistry of vaccination-site and nonvaccination-site sarcomas from cats in New Zealand," The New Zealand Veterinary Journal, vol. 55, no. 5, pp. 203-207, 2007.

[10] F. D. Doddy, L. T. Glickman, N. W. Glickman, and E. B. Janovitz, "Feline fibrosarcomas at vaccination sites and nonvaccination sites," Journal of Comparative Pathology, vol. 114, no. 2, pp. 165-174, 1996.

[11] S. S. Couto, S. M. Griffey, P. C. Duarte, and B. R. Madewell, "Feline vaccine-associated fibrosarcoma: morphologic distinctions," Veterinary Pathology, vol. 39, no. 1, pp. 33-41, 2002.

[12] Vaccine-Associated Feline Sarcoma Task Force, "VaccineAssociated Feline Sarcoma Task Force guidelines. Diagnosis and treatment of suspected sarcomas," Journal of the American Veterinary Medical Association, vol. 214, no. 12, p. 1745, 1999. 


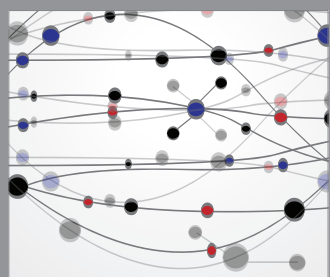

The Scientific World Journal
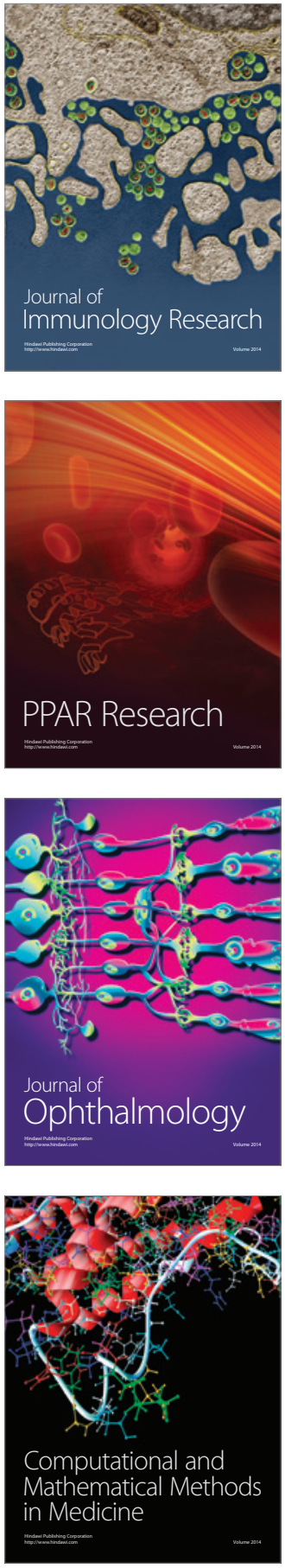

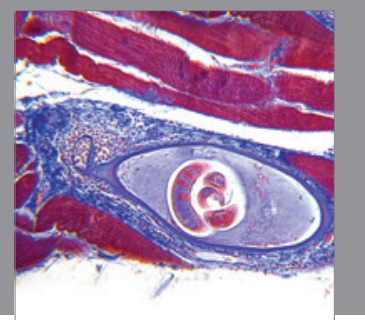

Gastroenterology

Research and Practice
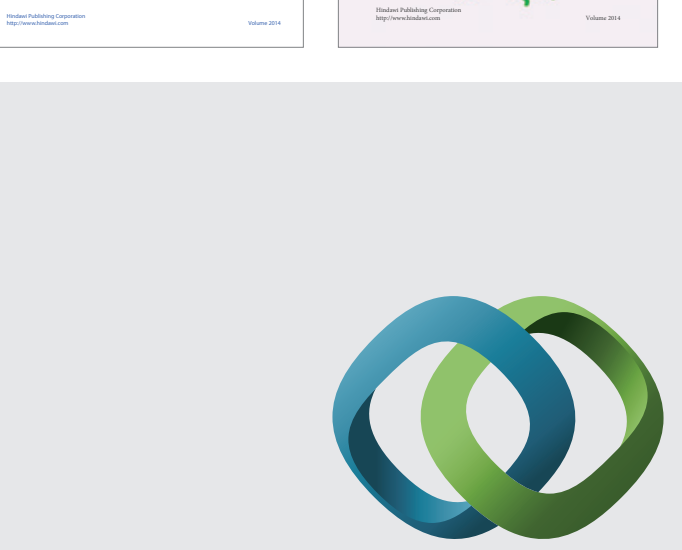

\section{Hindawi}

Submit your manuscripts at

http://www.hindawi.com
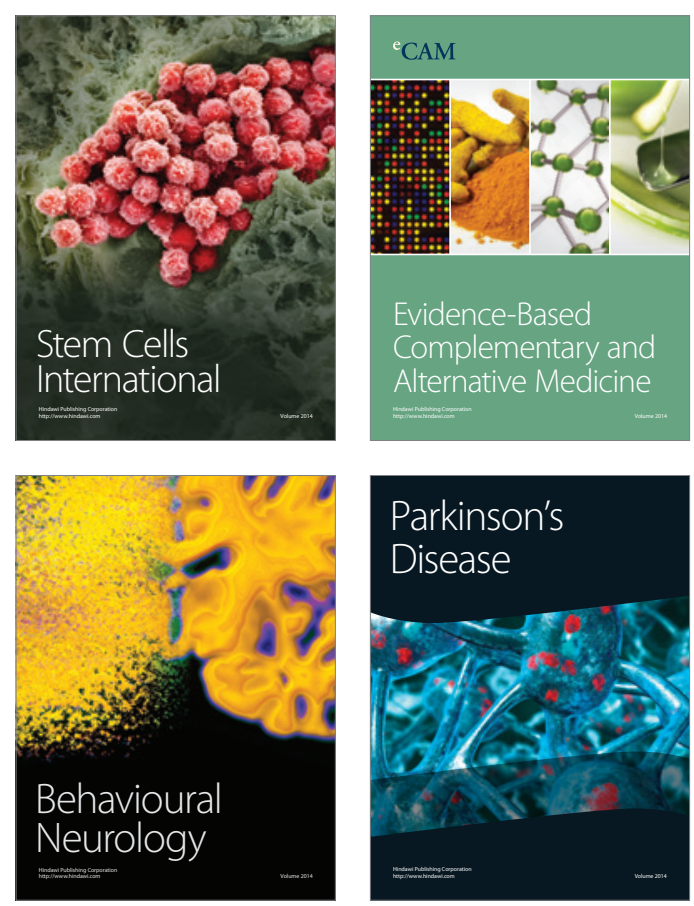

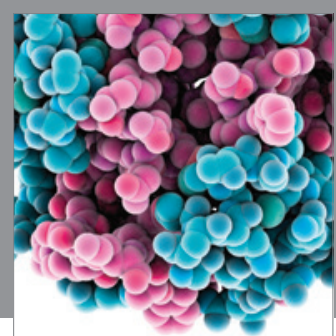

Journal of
Diabetes Research

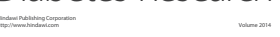

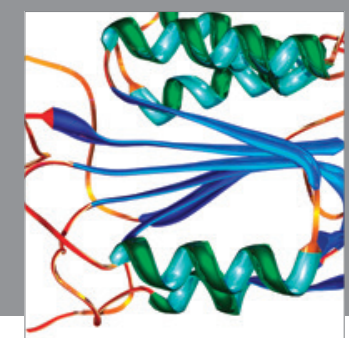

Disease Markers
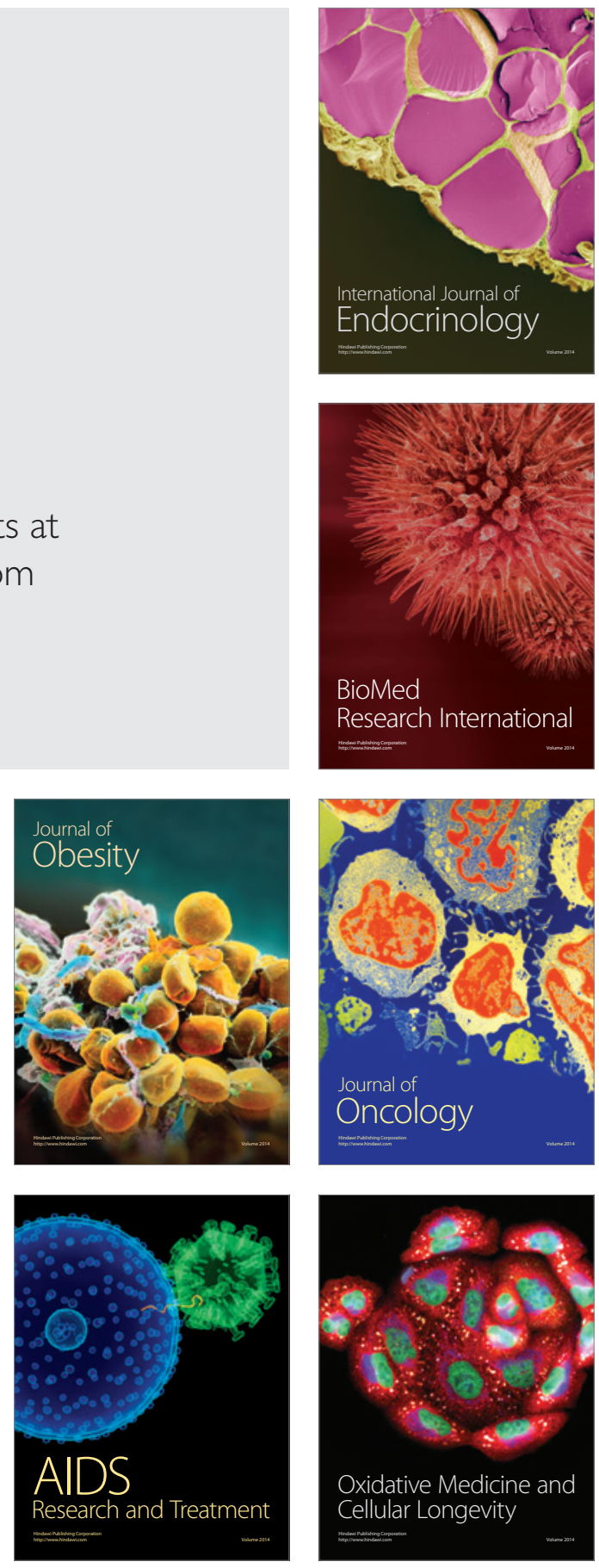